\title{
U.S. Geological Survey World Wide Web Information
}

The U.S. Geological Survey (USGS) invites you to explore an earth science virtual library of digital information, publications, and data. The USGS World Wide Web sites offer an array of information that reflects scientific research and monitoring programs conducted in the areas of natural hazards, environmental resources, and cartography. This list provides gateways to access a cross section of the digital information on the USGS World Wide Web sites.

\section{World Wide Web Sites}

\section{Primary Home Pages}

U.S. Geological Survey

www.usgs.gov

ASK USGS
ask.usgs.gov

USGS Search

search.usgs.gov

\section{USGS Library}

library.usgs.gov

Biological Resources Information biology.usgs.gov

Geologic Information
geology.usgs.gov

National Mapping Information mapping.usgs.gov

Water Resources Information water.usgs.gov

\section{Data and Information Sites}

Amphibians

www.frogweb.gov

Ask-a-Geologist

walrus.wr.usgs.gov/ask-a-geologist

Biological Cooperative Research Units biology.usgs.gov/coop
Biological Resources National Programs

biology.usgs.gov/pub_aff/natprog.html

Biological Science by State

biology.usgs.gov/state.partners/

partner.html

Biological Science and Technology Centers biology.usgs.gov/pub_aff/centers.html

Breeding Bird Survey

www.mp2-pwrc.usgs.gov/bbs

Butterflies of North America

www.npwrc.usgs.gov/resource/distr/

lepid/bflyusa/bflyusa.htm

Center for Integration of Natural Disaster Information

cindi.usgs.gov

Coastal and Marine Geology

marine.usgs.gov

Current Streamflow Conditions

water.usgs.gov/realtime.html

Daily Water Conditions

water.usgs.gov/dwc

Data Available from EROS Data Center edc.usgs.gov/dsprod/prod.html

Declassified Intelligence Satellite

Photographs

edc.usgs.gov/glis/hyper/guide/disp

Digital Backyard

mapping.usgs.gov/digitalbackyard

Earth Explorer

earthexplorer.usgs.gov

Earthquake Information

earthquake.usgs.gov

geohazards.cr.usgs.gov/earthquake.html

quake.wr.usgs.gov

wwwneic.cr.usgs.gov

Earth Science Information Center (ESIC) ask.usgs.gov
Earth Surface Dynamics

geochange.er.usgs.gov/esd

Energy Resources

energy.usgs.gov

EROS Data Center Home Page

edc.usgs.gov

Geographic Names Information System (GNIS)

geonames.usgs.gov

Ground Water Information

water.usgs.gov/ogw

Historical Streamflow

waterdata.us.gs.gov

Landsat7 Remote Sensing Satellite Data landsat7.usgs.gov

Landslide Information

landslides.usgs.gov

Locating Publications and Data Products www.usgs.gov/pubprod/index.html\#online

Mineral Resources

minerals.usgs.gov

Mineral Resources On-Line Spatial Data mrdata.usgs.gov

National Geologic Mapping

ncgmp.usgs.gov

National Geospatial Data Clearinghouse, USGS Node

nsdi.usgs.gov

National Satellite Land Remote Sensing Data Archive

edc.usgs.gov/programs/NSLRSDA.html

National Water Conditions

water.usgs.gov/nwc

National Water-Quality Assessment

Program

water.usgs.gov/nawqa 
National Water Research Program

water.usgs.gov/nrp

National Wetlands Research Center

www.nwrc.usgs.gov

North American Reporting Center for Amphibian Malformations

www.npwrc.usgs.gov/narcam

Ordering Information

mapping.usgs.gov/esic/prices/

ordering_info.html

Pricing Information for USGS Products mapping.usgs.gov/esic/prices

Search Publications of the USGS

usgs-georef.cos.com

Toxic Substances Hydrology

toxics.usgs.gov/toxics

USGS Geographic Data Download edc.usgs.gov/doc/edchome/ndcdb/ ndcdb.html

USGS by Theme-Environment, Resources, Hazards, Information Management

www.usgs.gov/themes

USGS Fact Sheets

water.usgs.gov/wid/indexlist.html

USGS Information Products about Mapping and Related Subjects mac.usgs.gov/mac/isb/pubs/pubslists/

USGS State District Chiefs

water.usgs.gov/staterep.html

USGS Topographic Maps

mcmcweb.er.usgs.gov/topomaps

View USGS Maps and Aerial Photo Images Online

mapping.usgs.gov/partners/

viewonline.html

Volcano Hazards

volcanoes.usgs.gov

Water Data

water.usgs.gov/data.html

Water Use Data

water.usgs.gov/watuse

\section{Educational Sites}

Audience Track for Teachers and Students www.usgs.gov/tracks/teachers.html

Earthshots: Satellite Images of
Environmental Change

edcwww.cr.usgs.gov/earthshots/slow/

tableofcontents

Finding Your Way with Map and Compass mac.usgs.gov/mac/isb/pubs/factsheets/ fs03301.html

Kids Corner in Biology

biology.usgs.gov/features/kidscorner

\section{National Mapping Program Educational \\ Materials \\ mac.usgs.gov/mac/isb/pubs/pubslists/ edu.html}

National Wetlands Research Center

Education and Training

www.nwrc.usgs.gov/educ_out.html

The Learning Web: K-12 Education www.usgs.gov/education

Water Education Resources water.usgs.gov/education.html

\section{Other Sites}

Microsoft TerraServer

terraserver.microsoft.com

National Atlas of the United States nationalatlas.gov

National Biological Information

Infrastructure

www.nbii.gov

National Biological Information

Infrastructure Postcards

www.nbii.gov/datainfo/postcard

Postcards from the Prairie www.npwrc.usgs.gov/postcards/ postcards.htm

Water Science for Schools

ga.water.usgs.gov/edu

\section{Doing Business with the USGS}

Biological Resources Division Partnerships biology.usgs.gov/partnership

Contract and Grant Information

www.usgs.gov/contracts/

Enhancing Integrated Science

www.usgs.gov/integrated_science

Mapping Cooperative Research and

Development Agreements

mapping.usgs.gov/www/crada/crada.html mapping.usgs.gov/www/partners

Memorandums of Understanding with Other Federal Agencies

www.usgs.gov/mou

National Mapping Business Partner

Program

mapping.usgs.gov/www/partners/

bpmain.html

Technology Transfer Information www.usgs.gov/tech-transfer/

USGS Activities Related to American Indians and Alaska Natives www.usgs.gov/indian

USGS Partnerships www.usgs.gov/partnerships.html

\section{Employment Sites}

\section{Employment With the USGS}

www.usgs.gov/ohr

USGS Student Jobs

www.usgs.gov/student

USGS Vacancy Notification www.usgs.gov/ohr/notify.html

\section{Additional Information}

For information on other USGS products and services, call 1-888-ASK-USGS, use the Ask.USGS fax service, which is available 24 hours a day at 703-648-4888, or visit the general interest publications Web site on mapping, geography, and related topics at mac.usgs.gov/mac/isb/pubs/pubslist/ index.html.

For additional information, visit the ask.usgs.gov Web site or the USGS home page at www.usgs.gov. 